\title{
Case of Breast Carcinoma With Malignant Pleural Effusion Treated With Intrapleural Heated Cisplatin Mitomycin Chemotherapy After Pleurectomy
}

\author{
Tulay AKMAN ${ }^{1}$, Semiha HAVAY ${ }^{2}$, Dogan KOCA ${ }^{3}$, Olcun U. UNAL ${ }^{4}$, Ahmet ONEN ${ }^{2}$, Ahmet U. YILMAZ \\ ${ }^{1}$ İzmir Tepecik Education and Research Hospital, Department of Medical Oncology, Izmir \\ ${ }^{2}$ Dokuz Eylul Unıversity Medical Faculty, Department of Internal Medicine, Izmir \\ ${ }^{3}$ Istanbul Hospital, Division of Medical Oncology, Van, Turkey \\ ${ }^{4}$ Atatürk University Medical Faculty, Department of Medical Oncology, Erzurum \\ ${ }^{5}$ Dokuz Eylul Unıversity Medical Faculty, Department of Thoracic Surgery, Izmir \\ ${ }^{6}$ Izmir University Medical Park Hospital, Department of Medical Oncology, Izmir, TURKEY
}

To the Editor,

A 60-year-old female patient, who was diagnosed with invasive micropapillary carcinoma [Estrogen receptor (ER):+, Progesterone receptor (PR):+, CerB2:+] 10 years ago, was treated with hormone therapy and being followed-up for stable disease, presented to our clinic with the complaint of shortness of breath. During her physical examination, we detected a decrease of respiratory sounds in the right hemithorax. On the Positron Emission Computer Tomography (PET-CT), we observed right pleural diffuse effusion, metastatic thickening areas in the pleura of the right lower lobe's anterior and posterior segments and increased CA 15-3 level. The patient, whose pleural fluid sampling gave a result consistent with malignant cytology, underwent chemical talk pleuredesis following closed tube drainage using tube thoracostomy. The patient received three cycles of systemic chemotherapy with paclitaxel. However, upon the persistence of the resistant pleural effusion, the patient was consulted in collaboration with the Department of Thoracic Surgery. The patient underwent partial pleural biopsy, decortication operation and intraoperative hot chemotherapy with cisplatine $200 \mathrm{mg}$ and mitomycin $20 \mathrm{mg}$ was given along with thoracotomy. The pathological features of the pleural biopsy were consistent with metastatic breast carcinoma. Immunohistochemical staning was positive for ER, PR and Cerb-B2 (Figure 1). The patient with marked response on post-operative PET-CT imaging started to receive anastrozol $1 \mathrm{mg} /$ day. The patient is monitorized as a case of stable disease and high performance during 48-months post-operative period.

More than $75 \%$ of the malignant pleural effusions result from lung, breast, ovarian carcinomas and lymphomas. ${ }^{1}$ Malignant pleural effusions may directly result from pleural infiltration by cancer cells or may be indirectly caused by mediastinal lymph node obstruction, bronchial obstruction, pulmonary thromboembolism, vena cava superior syndrome and decreased oncotic pressure.

In breast carcinoma, direct dissemination via chest wall may be seen or it may be due to metastatic involvement of the pleura. $^{2}$ In less than $30 \%$ of the patients who develop malignant pleural effusion related to breast carcinoma, symptom control may be achieved through chemotherapy and hormonal therapy. ${ }^{3}$ Conventional therapies include repeated thoracentheses, closed thoracotomy, pleuredesis, intrapleural chemotherapy with cytotoxic chemotherapeutic agents and pleurectomy. ${ }^{4}$ Unlike traditional sclerosing agents, intrapleural chemotherapy may potentially treat the underlying malignancy as well as pleural effusion. ${ }^{5}$ It has been shown that hyperthermia increase the cytotoxicity of many chemotherapeutic agents in human cell culture models and animal models. ${ }^{6}$ Although there are lots of clinical trials evaluating the heated intraperitoneal chemotherapy, there are limited data about heated intrapleural chemotherapy in malign pleural effusions. ${ }^{7}$ 


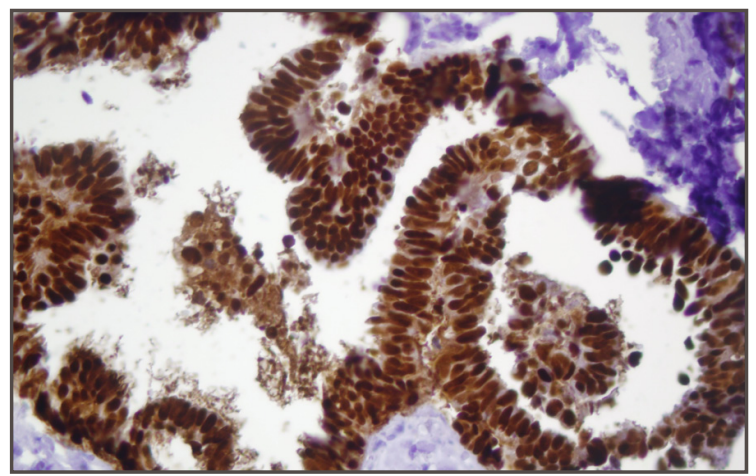

Figure 1. ER-positive tumor cells shown using immunohistochemical staining

But the results of these limited number of trials are promising. They showed that heated intrapleural chemotherapy in combination with cytoreduction provided significantly better survival. ${ }^{8}$ Cytarabine, etoposide, fluorouracil and mitomycin are other agents used for intrapleural chemotherapy. ${ }^{9}$ Cisplatine was demonstrated to be efficient and safe in intrapleural chemotherapy. Although there are lots of agents that can be administrated with different dosages for heated intrapleural chemotherapy, cisplatin is the most preferred agent due to its direct cytotoxic activity. ${ }^{10}$ Several toxic side effects have been reported in previous studies related to intrapleural cisplatin. In a study which 46 patients with metastatic malignant pleural effusions were evaluated, they reported grade 3 renal toxicity in 4 patients, grade 3 hematologic toxicity in 4 patients and grade 3 cardiopulmonary toxicities in 5 patients. ${ }^{3}$ Although cisplatin has number of side effects that limit its use in heated intrapleural chemotherapy, major concern is nephrotoxicity of intrapleural heated chemotherapy. But nephrotoxicity can be succesfully managed by intravenous fluid treatment without any complications. ${ }^{8}$

Consequently, although intrapleural heated therapy is not a commonly used method in the breast cancer patients with malignant pleural effusion, it may be an option in the treatment of resistant pleural effusion. It may be more efficient than systemic chemotherapy due to its direct passage to pleural cavity. However, the studies about this type of application are limited and new studies are warranted.

\section{REFERENCES}

1. Ismail-Khan R, Robinson LA, Williams CC Jr, et al. Malignant pleural mesothelioma: a comprehensive review. Cancer Control 13:255-263,2006.

2. Sahn SA. State of the art. The pleura. Am Rev Respir Dis 138:184-234,1988.

3. Figlin R, Mendoza E, Piantadosi S, Rusch V. Intrapleural chemotherapy without pleurodesis for malignant pleural effusions. LCSG Trial 861. Chest 106:363-366,1994.

4. American Thoracic Society. Management of malignant pleural effusions. Am J Respir Crit Care Med 162:1987-2001,2000.

5. Rusch WW, Figlin R, Godwin D, Piantadosi S. Intrapleural cisplatin and cytarabine in the management of malignant pleural effusions: a Lung Cancer Study Group trial. J Clin Oncol 9:313-319,1991.

6. Xu MJ, Alberts DS, Liu R, et al. In vitro evaluation of cisplatin interaction with doxorubicin or 4-hydroperoxycyclophosphamide against human gynecologic cancer cell lines. Cancer Chemother Pharmacol 25:89-94,1989.

7. Shigemura N, Akashi A, Ohta M, Matsuda H. Combined surgery of intrapleural perfusion hyperthermic chemotherapy and panpleuropneumonectomy for lung cancer with advanced pleural spread: a pilot study. Interact Cardiovasc Thorac Surg 2:671-675,2003.

8. Isik AF, Sanli M, Yilmaz M, et al. Intrapleural hyperthermic perfusion chemotherapy in subjects with metastatic pleural malignancies. Respir Med 107:762-767,2013.

9. Markman M, Cleary S, Pfeifle C, Howell SB. Cisplatin administered by the intracavitary route as treatment for malignant mesothelioma. Cancer 58:18-21,1986.

10. de Bree E, van Ruth $S$, Baas $P$, et al. Cytoreductive surgery and intraoperative hyperthermic intrathoracic chemotherapy in patients with malignant pleural mesothelioma or pleural metastases of thymoma. Chest 121:480-487,2002.

\section{Correspondence}

Dr. Tulay AKMAN

Tepecik Egitim ve Arastirma Hastanesi

Tibbi Onkoloji Bolumu

Gaziler Caddesi, No: 468

Yenisehir, IZMIR / TURKEY

Tel: (+90 232) 4443560

Fax: (+90 232) 4330756

e-mail: tulaytuzel@gmail.com 